\title{
Экспериментальное исследование усиления спонтанной эмиссии в микрорезонаторах на основе таммовских плазмонов с органической активной областью
}

\author{
(C) К.M. Морозов ${ }^{1,2}$, К.A. Иванов 2, , N. Selenin ${ }^{3}$, S. Mikhrin ${ }^{3}$, \\ D. de Sa Pereira ${ }^{4}$, C. Menelaou ${ }^{4}$, A.P. Monkman ${ }^{4}$, M.A. Калитеевский ${ }^{1,2,5}$ \\ ${ }^{1}$ Санкт-Петербургский Академический университет, \\ 194021 Санкт-Петербург, Россия \\ 2 Университет ИТМО, \\ 197101 Санкт-Петербург, Россия \\ ${ }^{3}$ Innolume $\mathrm{GmbH}$, \\ D-44263 Dortmund, Germany \\ ${ }^{4}$ Physics Department, Durham University, \\ Durham DH1 3LE, United Kingdom \\ ${ }_{5}^{5}$ Физико-технический институт им. А.Ф. Иоффе Российской академии наук, \\ 194021 Санкт-Петербург, Россия \\ 『 E-mail: kivanov1992@gmail.com, morzconst@gmail.com
}

(Получена 25 апреля 2018 г. Принята к печати 7 мая 2018 г.)

\begin{abstract}
Проведено теоретическое и экспериментальное исследование усиления спонтанной эмиссии излучения в микрорезонаторе на основе таммовского плазмона с активной областью из органического материала 4.4'-Bis $(N$-carbazolyl)-1.1'-biphenyl (CBP). Микрорезонатор состоял из брэгговского отражателя из оксида кремния и оксида тантала, поверх которого были нанесены слои СВР и серебра. Были рассчитаны зависимости модального фактора Парселла от направления излучения и частоты, а также спектр фактора Парселла. Были измерены спектры излучения и затухания флуоресценции во времени в ультрафиолетовом диапазоне. Установлено, что на частотах, соответствующих собственным модам таммовского плазмона, вероятность спонтанной эмиссии увеличивается, максимальное значение фактора Парселла достигает трех.
\end{abstract}

DOI: $10.21883 /$ FTP.2018.11.46589.11

\section{1. Введение}

Таммовские плазмоны (ТП) - состояние электромагнитного поля, локализованного на границе металла и брэгговского отражателя, недавно были предсказаны теоретически $[1,2]$ и экспериментально продемонстрированы [3,4]. В структурах на основе таммовских плазмонов были продемонстрированы макроскопическая оптическая когерентность [5], лазерная генерация [6] и излучение одиночных фотонов [7]. Недавно также была продемонстрирована возможность увеличения скорости спонтанной эмиссии при взаимодействии излучателя с таммовским плазмоном [8], несмотря на поглощение света в металле $[9,10]$. Цель данной работы исследование влияния таммовских плазмонов на скорость эмиссии излучения органическими материалами (4.4'-Bis $(N$-carbazolyl)-1.1'-biphenyl (CBP)), излучающими в ультрафиолетовом диапазоне [11].

\section{2. Изготовление образца и методика измерений}

На подложке кристаллического $\mathrm{SiO}_{2}$ методом магнетронного распыления был выращен четвертьволновый брэгговский отражатель в системе материалов $\mathrm{Ta}_{2} \mathrm{O}_{5} / \mathrm{SiO}_{2}$ (5 пар слоев, толщина слоя $\mathrm{Ta}_{2} \mathrm{O}_{5}$ составляет
43.2 нм, толщина слоя $\left.\mathrm{SiO}_{2}-66.4 \mathrm{Hм}\right)$ с центром фотонной запрещенной зоны на энергии 3.17 эВ.

Для дальнейшего создания микрорезонаторной структуры использовалась установка Kurt. J. Lesker Spectros, которая позволяет термически осаждать органические и неорганические материалы в виде тонких слоев при вакууме в $10^{-5}$ мбар. Осаждение 26 нм слоя СВР на готовую структуру $\mathrm{Ta}_{2} \mathrm{O}_{5} / \mathrm{SiO}_{2}$ распределенного брэгговского отражателя происходило со скоростью около $0.1 \mathrm{Hм} / \mathrm{c}$. Осаждение 50 нм серебра было осуществлено со скоростью около 0.05 нм $/$. Схема полученной структуры и ее фотография на электронном микроскопе продемонстрированы на рис. 1. Также был осажден слой материала СВР толщиной 26 нм на сапфировой подложке для сравнения с результатами, полученными при исследовании флуоресценции СВР, помещенного в микрорезонатор на основе таммовского плазмона.

Спектр флуоресценции слоя СВР при комнатной температуре (рис. 2) был получен с помощью спектрометра Jobin-Yvon Horiba Fluorolog FL3-22. Динамика затухания возбужденных состояний в изготовленной структуре измерялась с помощью метода, использующего счет одиночных фотонов с корреляцией по времени (Time Correlated Single Photon Counting, далее TCSPC). Данный метод основан на возбуждении исследуемой структуры импульсным лазером с дальнейшей регистрацией единичных фотонов флуоресцентного излучения и построе- 


\begin{tabular}{|c|}
\hline \multicolumn{1}{c}{} \\
\hline Silver \\
\hline $\mathrm{CBP}$ \\
\hline $\mathrm{SiO}_{2}$ \\
\hline $\mathrm{TaO}_{5}$ \\
\hline $\mathrm{SiO}_{2}$ \\
\hline $\mathrm{TaO}_{5}$ \\
\hline $\mathrm{SiO}_{2}$ \\
\hline $\mathrm{TaO}_{5}$ \\
\hline $\mathrm{SiO}_{2}$ \\
\hline $\mathrm{TaO}_{5}$ \\
\hline $\mathrm{SiO}_{2}$ \\
\hline $\mathrm{TaO}_{5}$ \\
\hline $\mathrm{SiO}_{2} \mathrm{substrate}$ \\
\hline
\end{tabular}

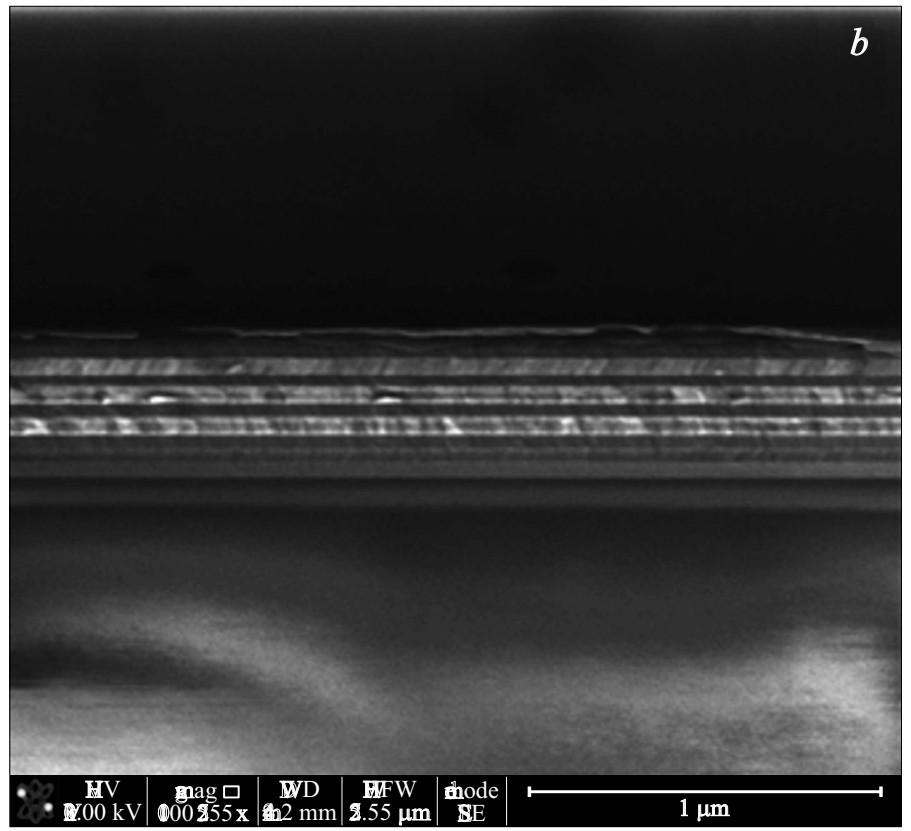

Рис. 1. $a-$ схема исследуемой таммовской структуры на основе распределенного брэгговского отражателя. $b-$ изображение скола исследуемой структуры, полученное с помощью электронного микроскопа.<smiles>c1ccc2c(c1)c1ccccc1n2-c1ccc(-c2ccc(-n3c4ccccc4c4ccccc43)cc2)cc1</smiles>

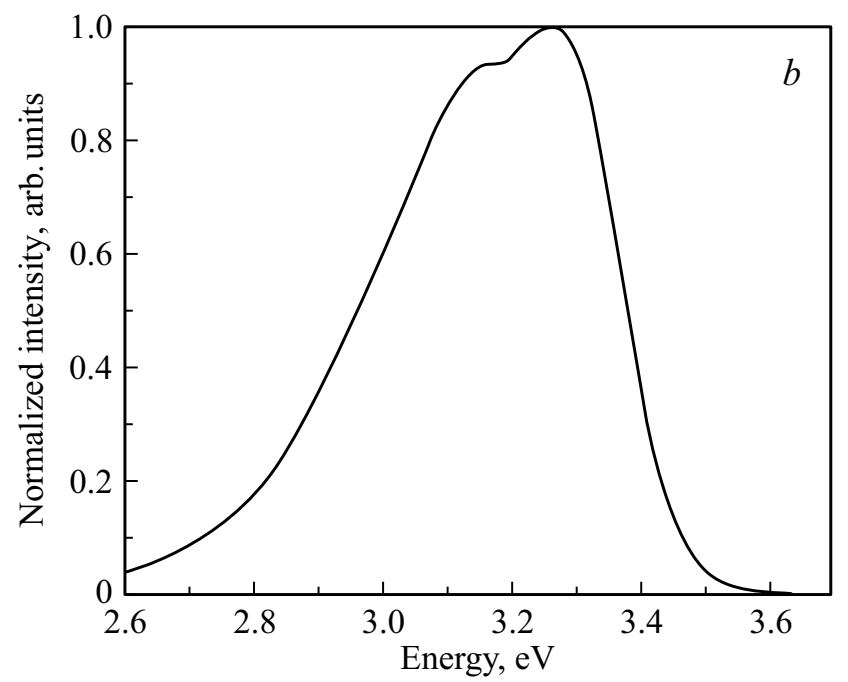

Рис. 2. Молекулярная структура $(a)$ и спектр флуоресценции (b) материала CBP (4.4'-Bis $(N$-carbazolyl)-1.1'-biphenyl), выполняющего роль активной области в таммовской структуре.

ния гистограммы плотности распределения фотонов во временно́й области [12].

В процессе измерения образец находился в вакууме при комнатной температуре. Возбуждение осуществлялось на длине волны 262 нм - удвоенная частота лазера длины волны 525 нм (область поглощения материала СВР) через слой серебра исследуемой микрорезонаторной структуры под углом в $45^{\circ}$. Регистрация флуоресценции от образца также осуществлялась под углом в $45^{\circ}$. Была проведена серия измерений на различных энергиях: 3.44, 3.35, 3.3 эВ, в области эмиссии СВР.

\section{3. Результаты и обсуждение}

Дизайн таммовской микрорезонаторной структуры был рассчитан таким образом, чтобы обеспечить соответствие частоты собственной моды таммовского плазмона для нормального распространения света максимуму спектра флуоресценции СВР (3.3 эВ). При таком выборе параметров структуры максимум спектра коэффициента Парселла располагается на частоте 3.35 эВ, на краю полосы спектра флуоресценции (рис. 3,a). Спектр коэффициента Парселла был рассчитан методом 

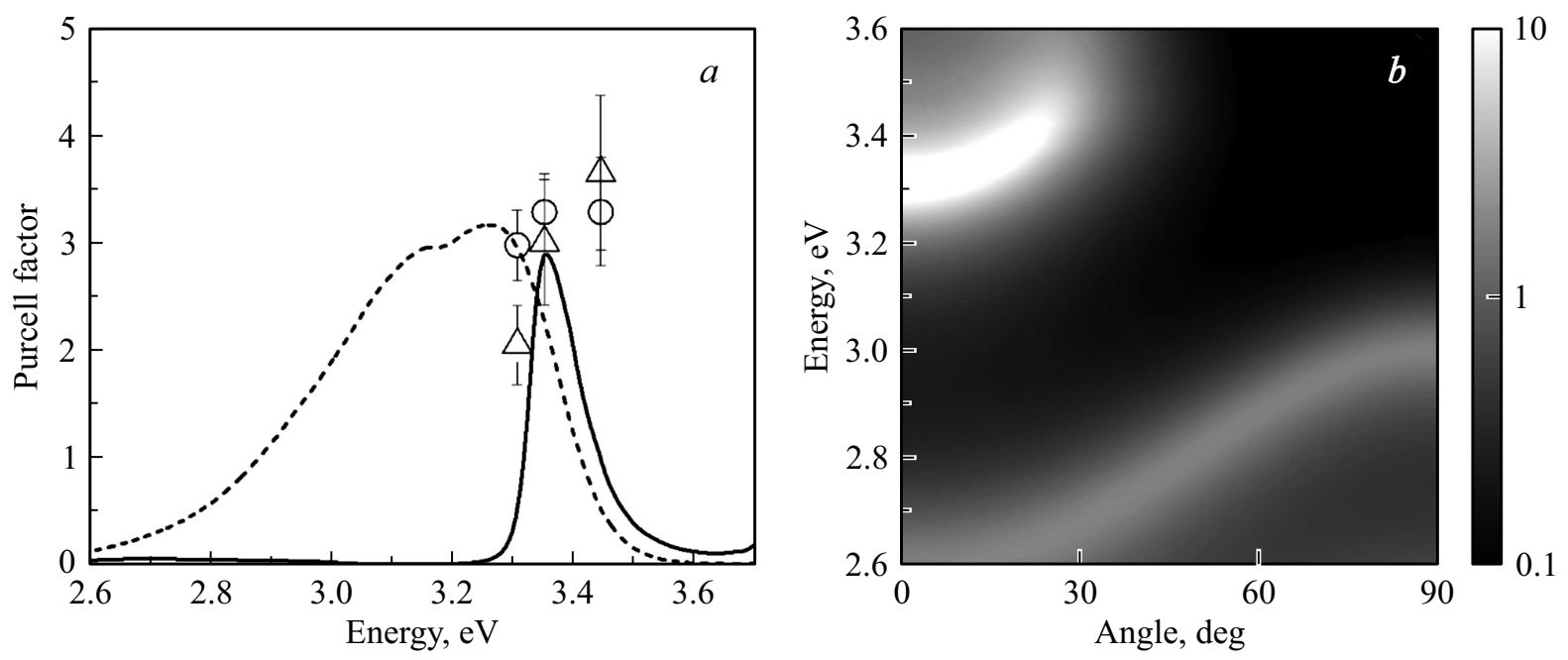

Рис. 3. $a-$ интегральный фактор Парселла, рассчитанный теоретически методом $S$-квантования (сплошная линия) для микрорезонатора на основе таммовского плазмона. Круги и треугольники показывают отношение обратных времен радиационного затухания для микрорезонатора на основе таммовского плазмона и СВР. $b$ - распределение модового фактора Парселла в зависимости от энергии и угла излучения.
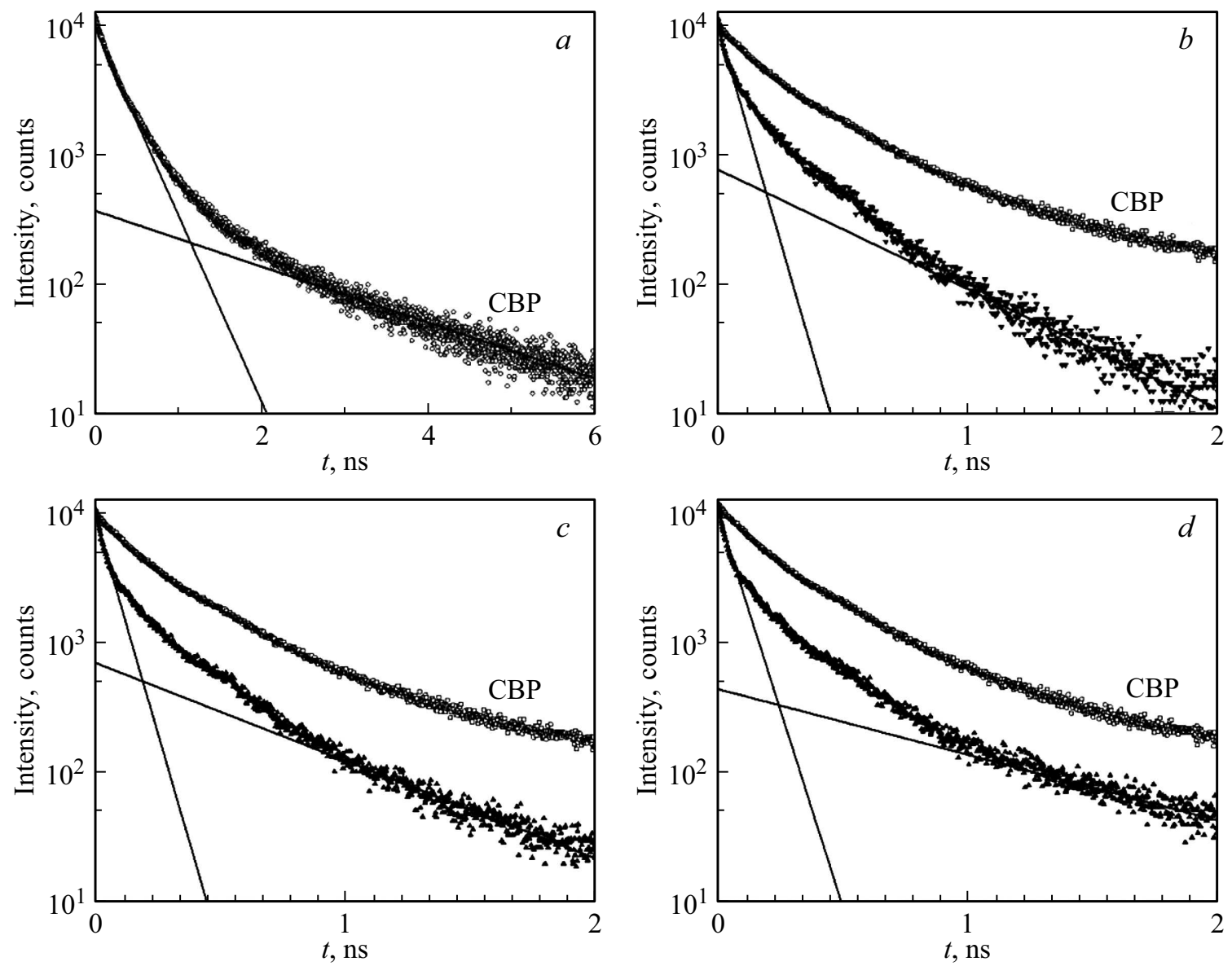

Рис. 4. Результаты временно-разрешающих экспериментальных измерений на основе методики TCSPC. $a-$ профиль затухания интенсивности флуоресценции от 26 нм слоя СВР на частоте 3.35 эВ. Профиль затухания интенсивности люминесценции микрорезонаторной таммовской структуры с СВР, соответствующими энергиям $3.44(b), 3.35(c)$, 3.3 эВ $(d)$. Для сравнения показаны области зависимости затухания люминесценции для одиночного слоя СВР. Прямые линии - результат аппроксимации экспоненциального затухания люминесценции в областях „быстрого“ и „медленного“ затухания. 
$S$-квантования [13]. На рис. 3, $b$ показана зависимость модального фактора Парселла (отношения плотности вероятности спонтанной эмиссии для излучателя в структуре и в свободном пространстве) [8,13]. Результаты измерений TCSPC в логарифмическом масштабе представлены на рис. 4. На рис. 4, $a$ показана динамика затухания люминесценции слоя СВР на подложке $\mathrm{Al}_{2} \mathrm{O}_{3}$ на частоте 3.35 эВ. Можно выделить две области: область „быстрого“ затухания и область „медленного“ затухания - данная зависимость является суммой двух затухающих экспонент. Время и быстрого, и медленного затухания примерно сохраняют свое значение во всей полосе люминесценции СВР.

На рис. $4, b-d$ представлены результаты, полученные при измерении люминесценции микрорезонатора, показанного на рис. 1, на частотах $3.44,3.35$ и $3,3.3$ эВ соответственно. Для сравнения показаны временны́е зависимости затухания фотолюминесценции СВР без резонатора. Видно, что по сравнению со случаем люминесценции органического эмиттера в свободное пространство интенсивность люминесценции затухает быстрее - время излучательной рекомбинации уменьшается, что является экспериментальным подтверждением существования эффекта Парселла в исследуемой таммовской структуре с органической активной областью. Можно заметить, что характер затухания также определяется двумя областями. Таким образом, экспериментально измеренный фактор Парселла - отношение обратных времен жизни возбужденных состояний - определяется также двумя областями.

На рис. 3, $а$ приведено сопоставление экспериментальных результатов (отношения обратных времен радиационного затухания в области быстрого затухания - кружки и в области медленного затухания — треугольники) с теоретически рассчитанным интегральным фактором Парселла (черная сплошная линия) - усредненным по всем углам эмиссии.

Для наглядности на рис. 3, $a$ также представлен спектр флуоресценции органического активного слоя (пунктирная линия).

В пределах погрешности эксперимента наблюдается четкая корреляция теоретической зависимости с экспериментом. Интересно отметить, что значения экспериментально определенных коэффициентов Парселла для быстрого и медленного участков затухания близки друг к другу и к теоретически рассчитанному значению коэффиента Парселла, что подтверждает радиационный характер релаксации носителей и на „медленном“, и на „быстром“ участках, а также то обстоятельство, что уменьшение времени жизни обусловлено эффектом Парссела. Необходимо также отметить, что экспериментально наблюдаемое уменьшение времени жизни несколько больше, чем теоретически рассчитанное. Данное обстоятельство, вероятно, обусловлено некоторым увеличением безызлучательной рекомбинации вблизи границ $\mathrm{CBP}$-серебро. Кроме этого, для СВР, помещенного в таммовскую структуру, изменяется спектр, спектры издучения, измеренные под разными углами, корелируют с модальным коэффициентом Парселла, показанным на рис. $3, b$.

\section{4. Заключение}

Проведено исследование изменения скорости спонтанной эмиссии излучения в ультрафиолетовой области спектра 4.4-Bis ( $N$-carbazolyl)-1.1'-biphenyl (CBP) при связи с таммовским плазмоном. Рассчитана зависимость модального и интегрального коэффициентов Парселла. Изготовлен микрорезонатор на основе брэгговского зеркала из оксида кремния и оксида тантала, серебряного металлического зеркала, и активной области из СВР. Теоретически показано и экспериментально установлено, что в рассмотренной структуре модальный фактор Парселла достигает значения десяти, а интегральный фактор Парселла — трех.

Авторы выражают благодарность Российскому научному фонду (проект № 16-12-10503) и Министерству образования и науки России (государственное задание № 16.9789.2017/БЧ).

\section{Список литературы}

[1] A.P. Vinogradov, A.V. Dorofeenko, S.G. Erokhin, M. Inoue, A.A. Lisyansky, A.M. Merzlikin, A.B. Granovsky. Phys. Rev. B, 74 (4), 045128 (2006).

[2] M.A. Kaliteevski, I. Iorsh, S. Brand, R.A. Abram, J.M. Chamberlain, A.V. Kavokin, I.A. Shelykh. Phys. Rev. B, 76, 165415 (2007).

[3] T. Goto, A.V. Dorofeenko, A.M. Merzlikin, A.V. Baryshev, A.P. Vinogradov, M. Inoue, A.A. Lisyansky, A.B. Granovsky. Phys. Rev. Lett., 101, 113902 (2008).

[4] M.E. Sasin, R.P. Seisyan, M.A. Kalitteevski, S. Brand, R.A. Abram, J.M. Chamberlain, A.Yu. Egorov, A.P. Vasil'ev, V.S. Mikhrin, A.V. Kavokin. Appl. Phys. Lett., 92 (25), 251112 (2008).

[5] R. Brückner, A.A. Zakhidov, R. Scholz, M. Sudzius, S.I. Hintschich, H. Fröb, V.G. Lyssenko, K. Leo. Nature Photonics, 6 (5), 322 (2012).

[6] C. Symonds, G. Lheureux, J.P. Hugonin, J.J. Greffet, J. Laverdant, G. Brucoli, A. Lemaitre, P. Senellart, J. Bellessa. Nano Lett., 13 (7), 3179 (2013).

[7] O. Gazzano, S. Michaelis de Vasconcellos, K. Gauthron, A. Lemaitre, P. Senellart. Appl. Phys. Lett., 100, 232111 (2012).

[8] A.R. Gubaydullin, C. Symonds, J. Bellessa, K.A. Ivanov, E.D. Kolykhalova, M.E. Sasin, A. Lemaitre, P. Senellart, G. Pozina, M.A. Kaliteevski. Sci. Rep., 7, 9014 (2017).

[9] J.B. Khurgin. Nature Nanotechnology, 10 (1), 2 (2015).

[10] S. Brand, R.A. Abram, M.A. Kaliteevski. Phys. Rev. B, 75 (3), 035102 (2007).

[11] R.S. Nobuyasu, Z.J. Ren, G.C. Griffiths, A.S. Batsanov, P. Data, S.K. Yan, A.P. Monkman, M.R. Bryce, F.B. Dias. Adv. Optical Mater., 4 (4), 597 (2016).

[12] L.M. Bollinger, G.E. Thomas. Rev. Sci. Instrum., 32, 1044 (1961). 
[13] М.А. Калитеевский, А.Р. Губайдуллин, К.А. Иванов, В.А. Мазлин. Опт. и спектр., 121 (3), 446 (2016).

Редактор Г.А. Оганесян

\section{Experimental investigation of spontaneous emission amplification in Tamm plasmon structures with organic active area}

K.M. Morozov 1,2, K.A. Ivanov ${ }^{2}$, N. Selenin ${ }^{3}$, S. Mikhrin ${ }^{3}$, D. de Sa Pereira ${ }^{4}$, C. Menelaou ${ }^{4}$, A.P. Monkman ${ }^{4}$, M.A. Kaliteevski ${ }^{1,2,5}$

${ }^{1}$ St.Petersburg Academic University, 194021 St. Petersburg, Russia

2 ITMO Univesity, 197101 St. Petersburg, Russia

3 Innolume $\mathrm{GmbH}$, D-44263 Dortmund, Germany

${ }^{4}$ Physics Department, Durham University, South Road, Durham, DH1 3LE, United Kingdom

${ }^{5}$ loffe Institute,

194021 St. Petersburg, Russia

Abstract We have experimentally studied phenomenon of amplification of spontaneous emission rate in Tamm plasmon based microcavity structure with organic material 4.4'-Bis $(N$-carbazolyl)-1.1'-biphenyl (CBP) as active layer. Investigated Tamm structure was based on 5 pairs $\mathrm{Ta}_{2} \mathrm{O}_{5} / \mathrm{SiO}_{2}$ Bragg reflector with thermally evaporated layers of CBP and silver on top of it. Dependencies of modal Purcell factor on emission direction and angle and Purcell factor spectrum was calculated. Decay dynamics and spectrum of fluorescence in UV range was measured. Fact that on frequencies corresponding to Tamm plasmon eigenfrequencies, increasing of spontaneous emission probability was demonstrated. Maximum value of Purcell factor reaches 3 . 